\title{
Correspondence
}

\section{Intubating conditions}

To the Editor:

During a rapid sequence induction, "Cricoid pressure," (the Sellick Manoeuvre) is used to prevent, or at least delay, regurgitation from the stomach to the pharnyx. When a mildly difficult intubation occurs, an assistant may be asked to move the upper trachea or the thyroid cartilage either posteriorly or laterally to improve visualisation of the larynx. Drs. Bruin and Buckley appear to equate the two quite different anatomical manipulations by referring to them both as "Cricoid pressure." This, I feel, only causes confusion in the minds of those nurses and technicians who assist us during induction.

\section{J.D. (Ian) St. Clair FFARCS \\ Edmonton, Alberta}

\section{$R E P L Y$}

We thank Dr. St. Clair for his response. The intent of our letter was to explain a mechanism by which well intentioned "Cricoid pressure" applied when the head is not in an incubating position, can interfere with, rather than facilitate, intubation during rapid sequence induction (RSI).

Because the cricoid cartilage is in intimate contact with both the proximal trachea and thyroid cartilage, pressure on it can be used to to manipulate the larynx into veiw during both RSI and difficult incubation. In order to improve visualisation during RSI the "cricoid" pressure should be applied while the head is maintained in the "sniffing" postion. If it is not, skin tension and pressure can cause the head to be fixed in flexion. Pressure may then need to be released and reapplied to improve visualisation, a move which could increase the risk of aspiration. By comparing the application and purpose of laryngeal pressure during RSI with that applied during difficult incubation assistants will be better able to help.

Norm Buckley MD FRCPC

Gerard Bruin MD FRCPC

Hamilton, Ontario

\section{Oesophageal aortic echo-Doppler and TEE}

To the Editor:

We read with interest the report by Gueugniaud et al. on continuous oesophageal echo-Doppler measurement of aortic blood flow. We wish to point out that, contrary to the author's claims, transoesophageal echocardiography (TEE) is readily available not only for infants but also for neonates. Paediatric biplane probes have been available since at least 1994 in North America and before that paediatric monoplane probes were used in neonates and infants. At Montréal Children's Hospital, we use biplane probes in neonates as small as $2.2 \mathrm{~kg}$. The TEE is not only used for cardiac procedures but also for neurosurgical and orthopaedic procedures or whenever additional cardiac monitoring is required for a patient. As paediatric TEE is now widely used, it would be of interest to compare it with the oesophageal device for both reliability and cost-benefit.

Josée Lavoie MD

Montréal Children's Hospital

Montreal, Quebec

\section{REFERENCE}

1 Gueugniaud P-Y, Mucbada R, Moussa M, Haro D, Petit $P$. Continuous oesophageal aortic blood flow echo-Doppler measurement during general anaesthesia in infants. Can J Anaesth 1997; 44: 745-50.

\section{REPLY}

We appreciate Dr Lavoie's interest in our article. ${ }^{1}$ The letter raises important precisions concerning the use of transoesophageal echocardiography (TEE) in infants and in neonates. In Canadian paediatric hospitals, paediatric TEE with trained operators seems to be now widely available for infants and for neonates whatever the surgical procedures. Unfortunately, it is not the case everywhere, even in some university hospitals in France.

This issue emphasises the interest of the echoDoppler aortic blood flow (ABF) device. However, it remains a subordinate reason for we agree that this new oesophageal echo-Doppler device could not take the place of TEE. The data provided by echocardiography allow a diagnosis of a cardiovascular status at a given moment during the perioperative period: they are accurate and irreplaceable for a momentary assessment, but discontinuous. In contrast, the $\mathrm{ABF}$ echoDoppler device is a monitor which, by integrating an haemodynamic profile, exhibits trends of the cardiovascular changes. This additional cardiac monitoring is an easy and completely operator-independent way to monitor continuously the haemodynamic variations during (paediatric) anaesthesia. As the findings on 
haemodynamic changes seem reliable and as this $A B F$ echo-Doppler device is greatly cheaper and easier to handle than a TEE device, we believe that this method of non-invasive continuous ABF measurement could be valuable for infants (and for adults) during anaesthesia in most general hospitals.

\section{P.-Y. Gueugniaud MD}

R. Muchada MD

Department of Araesthesiology

Edouard Herriot Hospital and Claude Bernard

University

Lyon, France

\section{RE FE RENCE}

1 Gueugnioud P-Y, Muchada R, Moussa M, Haro D, Petit $P$. Continuous oesophageal aortic blood flow echoDoppler measurement during general anaesthesia in infants. Can J Anaesth 1997; 44: 745-50.

\section{Modifications to the Bullard laryngoscope; when time is short}

To the Editor:

A number of modifications to the Bullard laryngoscope (BL) have been previously described which improved ease of use. ${ }^{1}$ As a result of reducing the terminal angulation of the dedicated intubating stylet (to $15^{\circ}$ ), the ETT is positioned more centrally in the glottis and rarely is the right arytenoid cartilage an obstruction to tube passage as has been previously reported. An oxygen tubing is attached to the luer lock of the BL suction port using a double male Luer-lok adapter (Figure I). Continuous oxygen insufflation through the port (6-10 L.min ${ }^{-1}$ flow) preserves patient oxygen saturation and both maintains the viewing aperture clear of secretions and clears fogging of the scope.

Further modifications have now been made and subsequent experience has been sufficiently positive to warrant mention. To adapt the BL for use with fibreoptic direct laryngoscope handles, the centre of the bulbring which holds the lightbulb when the BL is used with a standard handle light source was drilled out, removing the thread and increasing the ring opening from $3 \mathrm{~mm}$ to $5 \mathrm{~mm}$ in diameter. Using a stainless steel fibreoptic handle (Lyncs $\odot$ Rusch Inc.), the illumination provided is more than adequate for direct endoscopy but is not sufficient for operative video cameras. The weight of this configuration $(643 \mathrm{~g})$ can be further reduced to $484 \mathrm{gm}$ with the use of a fibreoptic penlight handle (Figure 2). Illumination with the penlight source is decreased compared to the full-sized battery handle but has been ade-

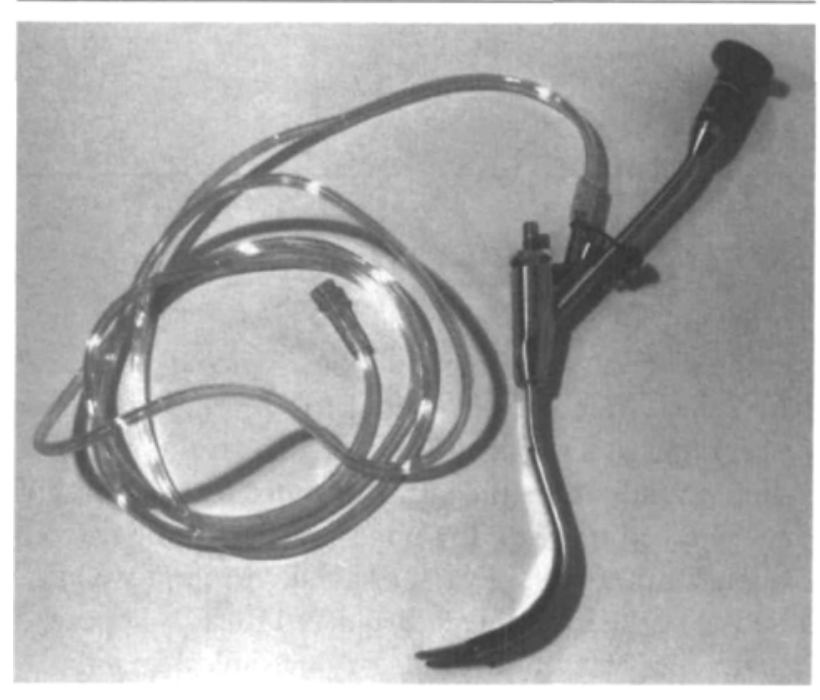

FIGURE 1 Oxygen tubing secured to BL with Luer-lok connection.

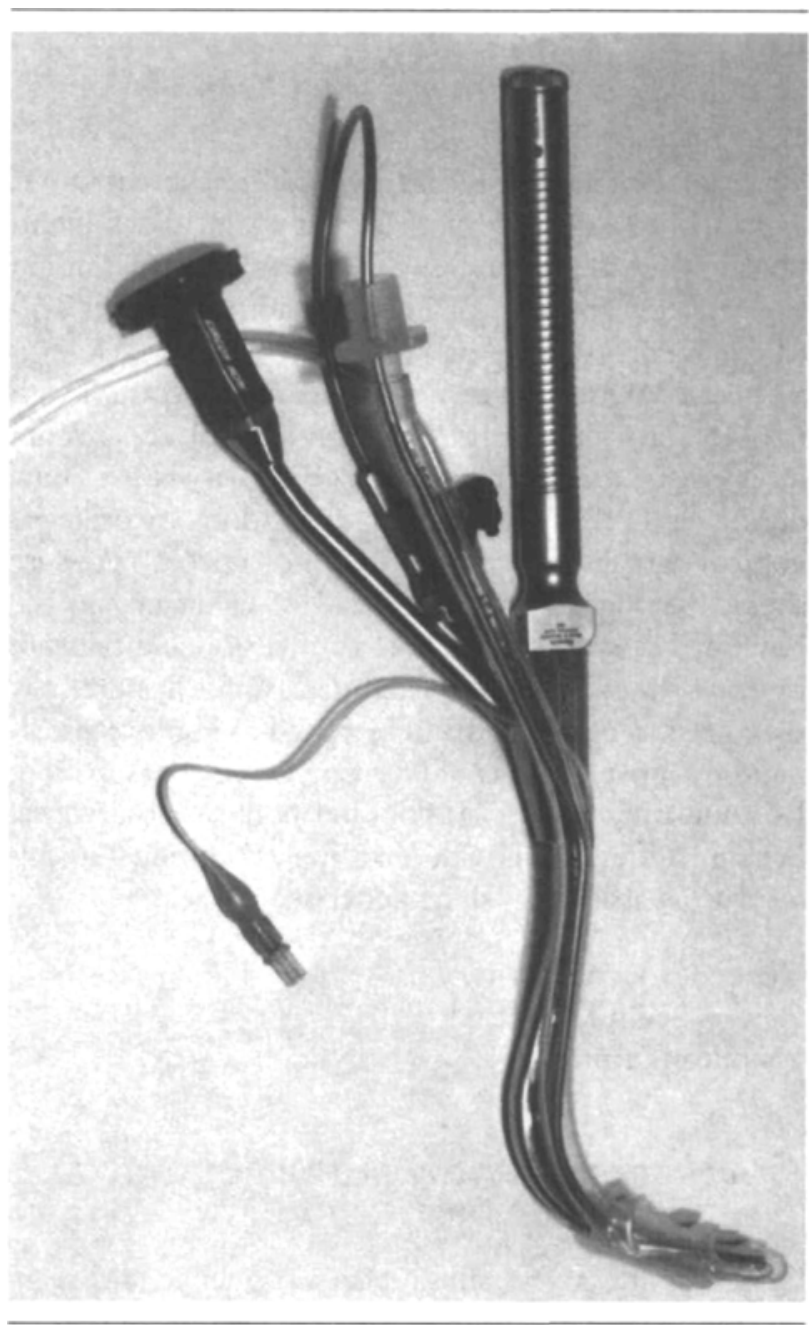

FIGURE 2 The BL configured with a fibreoptic penlight handle, oxygen rubing and an ETT mounted on the dedicated stylet. 